\title{
Ueber die Gestalt des Schweifes des grossen Cometen von 1882.
}

Da in der Abbildung zu meinem Bericht in Nr. 2468 die Figur des Schweifes sehr ungenügend hervortritt, werde ich jetzt in bestimmter Weise angeben, wie die bisher unbekannte Erscheinung von mir in der Zeit von Oct. 4 bis Nov. 2 I gesehen und jedesmal gezeichnet ward. Die unge- wöhnliche Gestaltung ist auch anderswo gesehen und auch wohl als ein gegen die Sonne gerichteter Schweif aufgefasst worden. Wie die Erscheinung in Wahrheit beschaffen war, will ich mit Hülfe der beigegebenen Abbildung darlegen.

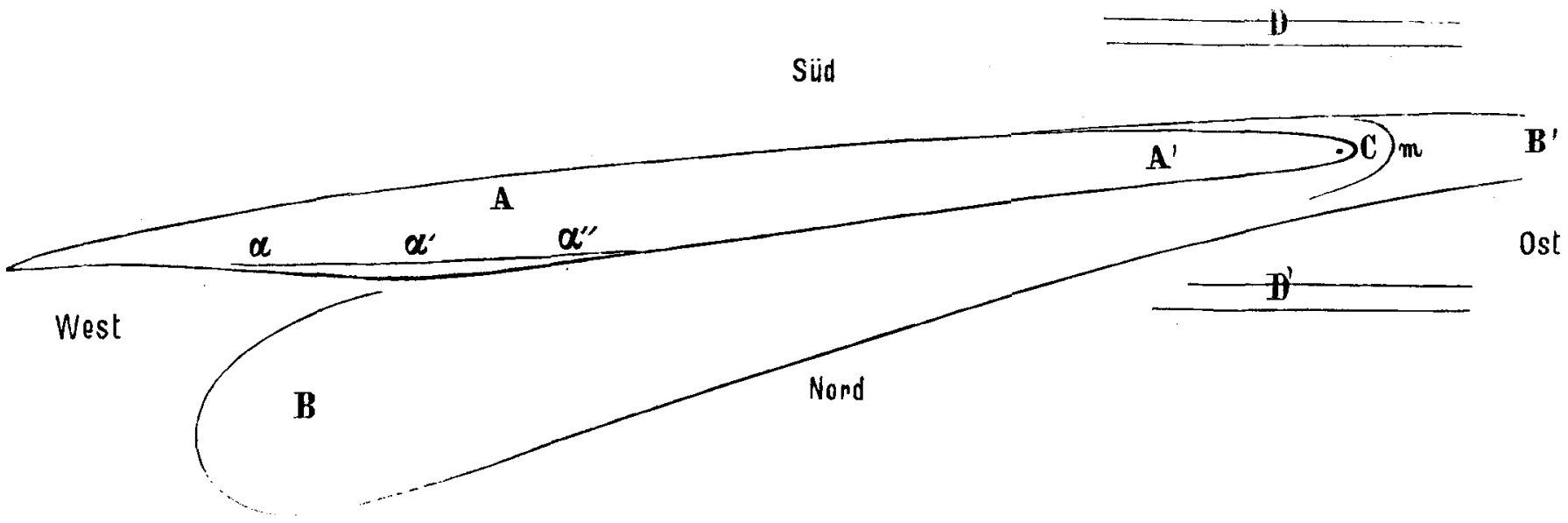

$A A^{\prime}$ ist der Hauptcomet, am Sucher bis $23^{\circ}$ lang, $B B^{\prime}$ die schwächere den Cometen umhüllende I,ichtmasse, die ich das Nebelrohr nenne, weil der Anblick, besonders in der Nähe von $C$, dem Kopfe des Cometen, einen andern Vergleich gar nicht aufkommen liess. Von $C$ bis $I^{\prime}$, also östlich über den Cometen hinaus, und gegen die Sonne hin, sah ich das $I^{\circ}$ breite Nebelrohr (bis Nov. 2 I) jedesmal auch mit freiem Auge, $I^{\circ}$ bis $2^{\circ}$ weit, wenn das Mondlicht nicht hinderte; $3^{\circ}$ bis $5^{\circ}$ dagegen am Sucher. Ausserdem gewahrte ich am Sucher und am Refractor, Oct. $7,8,9$, го, und später nicht wieder, den Halo $m$, aussserhalb der Region der gewöhnlichen Coma; er war mit dem Kerne des Cometen nahe concentrisch. Die beiderseitig mit den Seiten des Nebelrohres parallel gestellten geraden Nebelarme oder Säume $D D^{\prime}$ sah ich zuerst Oct. ro. Im Mondlicht schwanden sie viel früher als das Stück $B^{\prime}$. Nach Nov. 6 konnte ich $D$ und $D^{\prime}$ nicht ment mit Sicherheit erkennen. Seit Oct. I I war ich viel bemüht, für diese 2 Nebelarme den vermutheten abgerundeten tbschluss östlich rom Kerne zu entdecken, was mir indessen mit dem sehr schlechten Sucher in keiner Nacht gelungen ist. Eine Beobachtung Schiapparelli's Oct. I ) (mir damals brieflich mitgetheilt), wird später wohl zu erklären vermögen, wie diese Erscheinung aufzufassen sein müchte. Mir scheint es, dass sich im Perihele eine grossartige und plötzliche Ausströmung bildete, eine wahre Katastrophe, der zufolge der ä ussere Comet entstand, und an dem wir nur'die Reste bis $D D^{\prime}$, vielleicht auch das Nebelrohr, erkennen. Setzt man für Oct. 5 die Entfernung des Cometen von der Erde $=$ I (obgleich zu gering), so war der Durchmesser $D D^{\prime}$ $=8^{3} / 4$ Durchmesser der Mondbahn, oder ungefähr 873000 g. Meilen; der Durchmesser des Nebelrohrs bei $C=135000$ g. M.; der Durchmesser der Coma des Cometen nahe I00000 g. M. - Die dunkle Linie in der Axe des hellen Schweifes war Oct. 24 bereits undeutlich und später unsichtbar. Das westliche Ende der Schweiffigur zeigte am Sucher im Verlaufe von 5 Wochen sich in seinen allgemeinen Umrissen nicht stark veränderlich. Der wahre Schweif, also $A A^{\prime}$, die helle südliche Abtheilung, verlief als sehr lichtschwache schmale Spitze, und war an der N. Seite bei $\alpha \alpha^{\prime} \alpha^{\prime \prime}$ auffallend heller, als irgend ein benachbarter Theil. Aus $a^{\prime}$ ward seit Nov, 6 eine deutliche, im dortigen feinen Lichtnebel isolirte $1 / \mathbf{2}^{\circ}$ breite Wolke, deren Ort ich oft genau vermerkt habe. Das Westende des Nebelrohres $B B^{\prime}$ war von Oct. 4 bis Nov. 2 I jederzeit abgerundet, doch zuletzt sehr verwaschen und schwer in der Zeichnung darzustellen. In 25 vollständigen Abbildungen, rectificirt nach den Sternen einer seit Oct. 3 entworfenen Charte der bekannten Katalogsterne, sind die Oerter von $\alpha^{\prime}$ und von $B$, der Mitte des abgerundeten Theiles, angegeben. Indem ich diese Positionen aus den (für das Aeq. I 850 geltenden) Zeichnungen entnahm, wurden sie durch Curven dargestellt und ausgeglichen. Beiderlei Werthe werde ich hier mittheilen, denn sie werden zum ersten Male Veranlassung geben zu Untersuchungen über die Bahnbewegung besonderer Nebelmassen innerhalb des Schweifes oder an den Gränzen desselben.

In Folgendem bedeutet: $\alpha^{\prime}$ einen hellern Punkt im Saume, später eine Nebelwolke an der NW. Seite des Hauptschweifes; $B$ die Mitte der westlichen Abrundung des Nebelrohres. Zum Grunde liegt das Aequinox von $185^{\circ}$. 


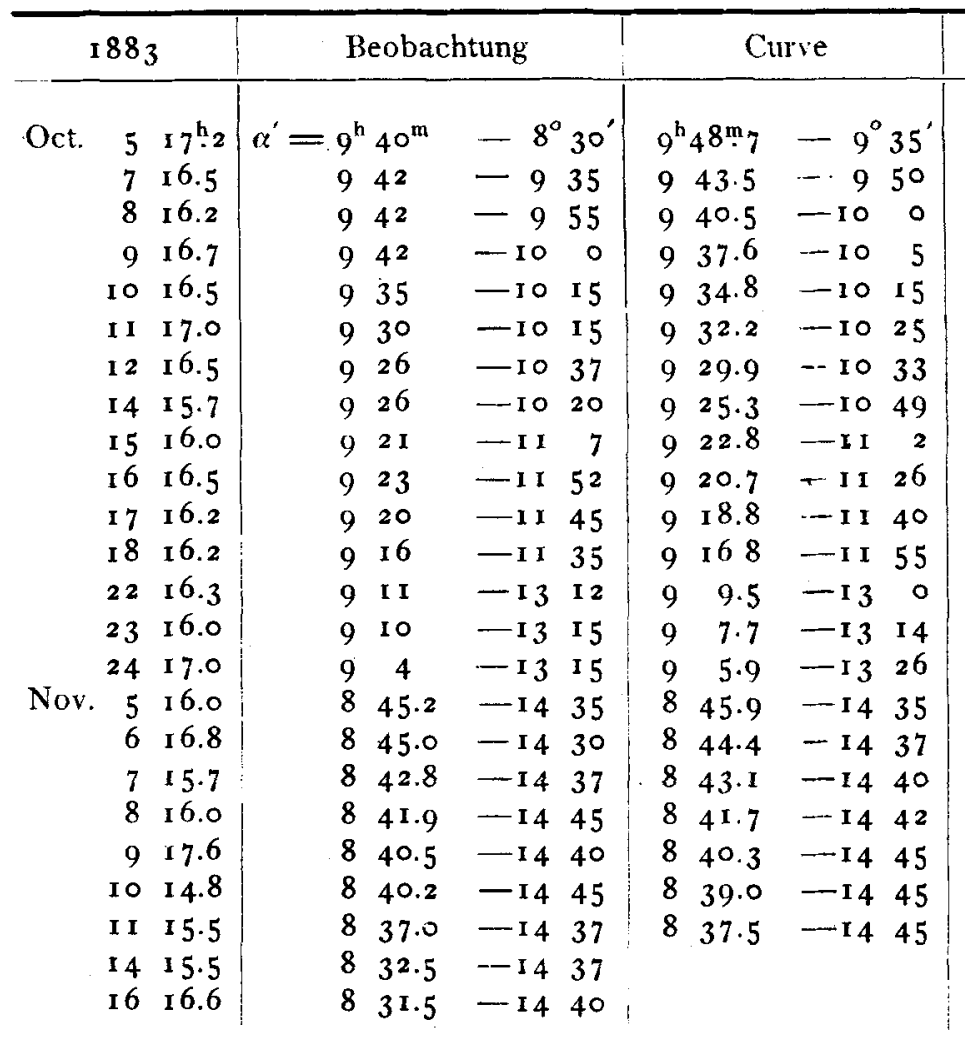

Die Angabe für Oct. 4 habe ich wegen des zll starken Mondlichtes gar nicht berücksichtigt. Für die folgende Rechnung ward auch Oct. 5 als noch zu unsicher ausgeschlossen.

Es wurde nun der jedesmalige Werth (Curve-Beob.) bestimmt, und für je 4 Gruppen sind die Angaben der Beob. mit den täglichen, der Curve entnommenen Bewegungen auf eine gewisse Zeit reducirt; sodann ward beiläufig der w. Fehler der Positionen berechnet.

$$
\alpha^{\prime} \text { (Curve-Beob.) } \quad B \text { (Curve-Beob.) }
$$

\begin{tabular}{|c|c|c|c|c|c|}
\hline \multirow[t]{15}{*}{ Oct. } & 5 & $--8 m \cdot 7$ & $-45^{\circ}$ & $+14^{m_{2}}$ & $-18^{\prime}$ \\
\hline & 7 & +1.5 & -15 & +1.3 & -10 \\
\hline & 8 & -1.5 & -5 & -2.5 & +10 \\
\hline & 9 & -4.4 & -5 & -1.0 & \\
\hline & 10 & -0.2 & 0 & +2.4 & -8 \\
\hline & I I & +2.3 & -10 & +1.0 & \\
\hline & 12 & +3.9 & +4 & +08 & +18 \\
\hline & 14 & -0.7 & -29 & -1.7 & -34 \\
\hline & 15 & +1.8 & +5 & +2.5 & +13 \\
\hline & 16 & --2.3 & +26 & -0.3 & \\
\hline & 17 & -1.2 & +5 & -1.0 & +18 \\
\hline & 18 & +0.8 & -20 & -0.5 & -2 \\
\hline & 22 & -1.5 & +12 & -2.2 & +9 \\
\hline & 23 & -2.3 & +1 & +0.2 & - \\
\hline & 24 & +1.9 & - II & +0.5 & - \\
\hline \multirow{3}{*}{ Nov. } & 5 & +0.7 & $\circ$ & - & - \\
\hline & 6 & -0.6 & -7 & +1.2 & +20 \\
\hline & 7 & +0.3 & -3 & +0.5 & - \\
\hline
\end{tabular}

Curve

$$
\begin{aligned}
& B=9^{h} 30^{m}-8^{\circ} 0^{\prime} \mid 9^{h} 44^{m} ._{2}-8^{\circ} 8^{\prime} \\
& 937-830 \quad 938.3-840 \\
& 93^{8}-9 \circ \quad 935.5 \cdots 850 \\
& 934-90 \quad 933.0-90
\end{aligned}
$$

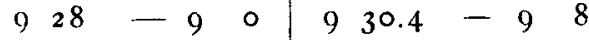

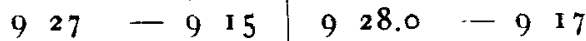

$$
\begin{aligned}
& \begin{array}{lllll|llll}
9 & 25 & -9 & 45 & 9 & 25.8 & -9 & 27
\end{array} \\
& 923-915 \quad 921.3-949 \\
& \begin{array}{llllllll}
9 & 17 & -10 & 15 & 9 & 19.5 & -10 & 2
\end{array}
\end{aligned}
$$

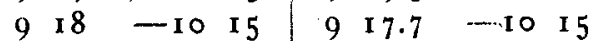

$$
\begin{aligned}
& \begin{array}{llll|llll}
9 & 17-10 & 45 & 9 & 16.0 & -10 & 27
\end{array} \\
& \begin{array}{llll|llll}
9 & \text { I } 5 & - \text { IO } & 35 & 9 & \text { I } 4.5 & - \text { I0 } 37
\end{array} \\
& \begin{array}{llll|llll}
9 & 10 & -11 & 30 & 9 & 7.8 & -11 & 21
\end{array} \\
& \begin{array}{llll|lllll}
9 & 6 & -1 \mathrm{I} & 30 & 9 & 6.2 & -1 \mathrm{I} & 3 \mathrm{I}
\end{array} \\
& \begin{array}{llllllll}
9 & 4 & \text {-I I } & 30 & 9 & 4.5 & \text {-II } & 39
\end{array} \\
& \begin{array}{l|rrr}
-\quad 3 & 847.9-1250
\end{array} \\
& \begin{array}{lllllll}
846 & -13 & 0 & 8 & 47.2 & -12 & 40
\end{array} \\
& \begin{array}{lll|llll}
846 & -12 & 30 & 8 & 46.5 & -12 & 36
\end{array} \\
& \begin{array}{llll|llll}
8 & 46 & -12 & 0 & 8 & 45.6 & \text { - I } 2 & 28
\end{array} \\
& \begin{array}{lll|llll}
844-12 & 0 & 8 & 448 & -12 & 20
\end{array} \\
& \begin{array}{llllllll}
844 & -12 & 30 & 8 & 44.0 & -12 & 10
\end{array}
\end{aligned}
$$

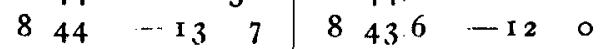

$$
\alpha^{\prime} \text { (Curve-Beob.) is (Curve-Beob). }
$$

$$
\begin{aligned}
& \text { Noy. } 8-0^{\mathrm{m}} \cdot 2+3^{\prime} \quad-0^{\mathrm{m}} \cdot 4-28^{\prime} \\
& 9-0.2-5+0.8-20 \\
& \begin{array}{rrrrr} 
& -1.2 & 0 & 0.0+20 \\
I I+0.5 & -8 & -0.4+67
\end{array}
\end{aligned}
$$

Für den hellsten Punkt $\alpha^{\prime}$ im Saume $\alpha \alpha^{\prime} \alpha^{\prime \prime}$ habe ich nun folgende Oerter (für 1 $85^{\circ}$ ) abgeleitet, wobei noch bemerkt sein mag, dass die Curvenwerthe für $18 \mathrm{Uhr} \mathrm{m} . Z$. Athen gelten, ebenso wie die neuen Mittelwerthe selbst.

$$
\alpha^{\prime}=
$$

Oct. 9 18.0 $9^{\mathrm{h}} 3^{8 \mathrm{~m}} \cdot 2 \mathrm{o}-9^{\circ} 5^{6: 5} 5$ B. Oct. $7,8,9,10, \mathrm{I}$ I

$$
\begin{aligned}
& \text { I } 5 \text { I } 8.0922 .72-\text { I I 8. I } 5 \geqslant \text { I } 2, \text { I 4, I 5, I 6, I } \\
& 2018.0913 .67-1225.35, \quad 17,18,22,23,24
\end{aligned}
$$

Nov. 9 I 8.0840 .53 - $433^{6.5} 6$ * Nov. $6,7,8,9$, 10, I 1

Ferner für $B$, das Westende des Nebelrohres.

$$
B=
$$

Oct. $9 \quad \mathbf{8} .0932 .80-857.05$ B. Oct. $7,8,9,10$, I I

$1518.0919 .72-1014.16,12,14,15,16,17,18$

2018.09 11.72-II $1.75 \geqslant 17,18,22,23,24$

Nov. 9 I8.0 844.64 - I 2 27.1 6 , Nov. 6, 7,8, 9, 10, I I

Zur beiläufigen Beurtheilung der Unsicherheit dienen die folgenden w. Fehler des Mittels 


\begin{tabular}{|c|c|c|c|c|c|c|}
\hline Oct. & $\alpha^{\prime} \ldots \pm$ & 0.98 & $\pm 2 \cdot 4$ & $B \ldots \pm$ & $\circ \stackrel{m}{6} 65$ & $\pm \quad 2: 8$ \\
\hline I 5 & \pm & 0.65 & \pm 5.7 & \pm & 0.35 & \pm 5.3 \\
\hline 20 & \pm & $0.6 \mathrm{I}$ & \pm 5.2 & \pm & 0.33 & \pm 3.7 \\
\hline Nov. 9 & \pm & O. 17 & +2.2 & \pm & 0.19 & \pm 9.5 \\
\hline
\end{tabular}

Oct. 9, 20, Nor. 9 sind also zur Bahnberechnung gut geeignet; für eine Vergleichung ausser der mittleren Position habe ich noch eine andere, die von Oct. I 5 , hinzugefügt.

Athen 1882 Nov. 23.

F. F. Ful. Schmidt.

\section{Auszug aus einem Schreiben von Prof. R. Wolf, Director der Sternwarte in Zürich, an den Herausgeber.}

Die soeben, in zweiten Hefte der Vierteljahrsschrift der naturf. Gesellschaft in Zürich pro 1882 , erhaltene No. 57 meiner Astronomischen Mittheilungen Linie eine Fortsetzung meiner Studien über die Sonnenfleckenperioden, in welcher ich den sichern Beweis geleistet zu haben glaube, dass sich in der Häufigkeit der Sonnenflecken, neben der mittlern Periode von etwas mehr als is $1 / 9$ Jahren, noch eine zweite Periode von nichtganz ro Jahren zeigt, welche sich mit der erstern combinirt, und dadurch die Schwankungen in der Länge der einzelnen Perioden erzeugt, - dass dagegen eine Periode in der Nähe von I2 Jahrenganz entschieden nicht existirt. Eine Versuchsreihe, welche ich zum Belege für die Richtigkeit eines bei dieser Untersuchung angewandten Grundsatzes anstellte, und ebenfalls in Nr. 57 mittheile, dürfte ebenfalls einen nicht unwichtigen Beitrag zu der Lehre von der Er- fahrungswahrscheinlichkeit darstellen. - Sodann theile ich die von meinem Assistenten, Herrn Wolfer, im zweiten Semester des letzten Jahres erhaltenen Sonnenflecken-Positionen mit, und schliesse mit einer kleinen Fortsetzung der Sonnenflecken-Literatur.

Der heutige Durchgang konnte wegen Wolken hier nicht beobachtet werden, trotzdem Alles in bester Bereitschaft war. Nur auf Augenblicke wurde Venus gesehen,einmal ungefähr in der Mitte zwischen den beiden Berührungen, und dann noch einmal etwas nach der innern Berührung. Immerhin konnte ich constatiren, dass die Vorausberechnung, welche ich vor ca. 2 Jahren machte, besser zutraf als andere der publicirten Zahlen. - Hoffentlich sind dafür die Beobachtungen an andern Orten gelungen.

Sternwarte Zürich 1882 Dec. 6.

\section{Meridian observation of the Great Comet of September 1882.}

Communicated by authority of Vice-Admiral Stephen C. Rowan U. S. N. Superintendent.

The following meridian observation of the Great Comet of $188 \mathrm{z}$ was obtained this morning by Assistant Astronomer Winlock, with the Transit Circle of the Naval Observatory:

$$
1882 \text { Nov. } 15.74 \text { RA: }=9^{h} 27^{m} 50^{s} .72 \quad \text { NPD. }=114^{\circ} 49^{\prime} \text { I } 8^{\prime \prime} .9 .
$$

The part observed wis the main point of condensation, near the following end of the nucleus.

The observation is corrected for refraction but not for parallax.

U. S. Naval Observatory, Washington 1882 Nov. I 6 .

A. V. Skinner, Assistant Astronomer, in charge 'Transit Circle.

\section{Osservazione del passaggio di Venere 1882 Dic. 6 a Roma.}

Jeri ad onta del tempo cattivo l'osservazione del passaggio di Venere riesci qui molto bene. Jo osservai i contatti collo spettroscopio a reticolo applicato all' equatoriale di Merz di $25^{\mathrm{cm}}$, e il Prof. Millosevich li osservó alla manièra ordinaria servendosi dell' equatoriale di Cauchoix di $15^{\mathrm{cm}}$, e con ingrandimento di $\mathrm{r} 30$.
Alle $2^{\mathrm{h}} 44^{\mathrm{m}} 33^{\mathrm{s}} \cdot 8$ tempo medio di Roma vidi il bordo del pianeta sulle punte delle fiamme cromosferiche, e dopo seguendo sempre distantamente l'avanzarsi di Venere, e notai il primo contatto esterno a

$$
2^{\mathrm{h}} 48^{\mathrm{m}} 54^{\mathrm{s}} \cdot 43
$$

